\title{
TRABAJO DE EQUIPO EN LAS ORGANIZACIONES. TRES INTERVENCIONES
}

\section{Teamwork in organizations: Three interventions}

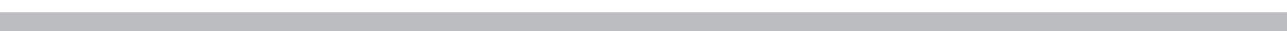

Arturo Solf Zárate*

Universidad Nacional Mayor de San Marcos - Lima, Perú https://orcid.org/0000-0003-3238-3504
\end{abstract}

\begin{abstract}
Resumen
Se presentan tres intervenciones para favorecer la labor de los equipos en las organizaciones: Desarrollo de Equipo, Construcción de Equipo, y Entrenamiento de Equipo. Motiva este trabajo la necesidad de precisar las diferencias entre dichas intervenciones. Está orientado a ser útil principalmente a estudiantes de la carrera de psicología. El documento incluye aspectos básicos desde una perspectiva práctica, y espera servir de estímulo para continuar aprendiendo sobre el tema, que actualmente presenta un extraordinario desarrollo.
\end{abstract}

Palabras claves: Trabajo de equipo, Desarrollo de equipo, Construcción de equipo, Entrenamiento de equipo, Roles del psicólogo, Capacitación, Organizaciones laborales.

\begin{abstract}
Three interventions are presented to promote teamwork in organizations: Team Development, Team Building, and Team Training. This study is motivated by the need to specify the differences between these interventions. It is aimed mainly at Psychology students. The document includes essential aspects from a practical perspective and intends to encourage students to continue learning on the subject, which is currently experiencing extraordinary development.
\end{abstract}

Keywords: teamwork, team development, team building, team training, psychologist roles, training, work organizations.

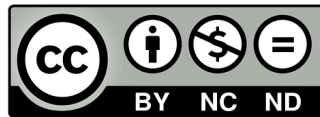

Cualquier uso que se haga de este artículo debe incluir: Autor / Título original de la publicación / ISSN

\footnotetext{
* Psicólogo. arturo.solf@gmail.com
} 


\section{INTRODUCCIÓN}

Actualmente el trabajo de equipo en las organizaciones es fundamental para el logro de sus metas, pero en la práctica se observa que muchos profesionales que proporcionan servicios para tener equipos más eficientes no poseen una clara diferenciación entre las siguientes intervenciones: Desarrollo de Equipo, Construcción de Equipo, y Entrenamiento de Equipo.

El presente trabajo pretende esclarecer los tres tipos de intervención, de manera que el profesional disponga de una adecuada fundamentación para su accionar y permita una mejor decisión para especializarse en el tema.

Otro motivo para la elaboración del presente documento es promover el interés para adquirir experticia en asesorar el trabajo de equipo, cuya temática ha logrado un desarrollo exponencial (Salas et al, 2017) con aplicaciones en rubros muy diversos, así un número especial de la APA sobre The science of teamwork (May- June 2018) incluye trabajos vinculados a contextos como: militar, colegios, hospitales, desastres, terrorismo. Los psicólogos actualmente están investigando para contribuir en la misión de astronautas que irán a Marte según los planes de la NASA; indagan cómo lograr que los tripulantes conformen un equipo de trabajo eficaz y eficiente (Paoletti, Kilcullen, \& Salas, 2021; Salas et al., 2015b).

El texto está orientado principalmente a estudiantes de la carrera de psicología y quizás pueda interesar a otros profesionales. Se adopta una perspectiva mayormente práctica y destaca lo básico de modo que permita un desarrollo posterior.

A continuación, se presenta definiciones y características de cada intervención. Su complementariedad. Orientaciones para un adecuado apoyo. Comentarios sobre la Construcción de Equipos Virtuales. Recomendaciones. Finalmente, en anexos se incluyen algunos ejemplos y recursos para precisar mejor las características de cada intervención.

\section{DESARROLLO DE EQUIPO}

Considere la siguiente situación:

Caso 1. "Para el ingreso de nuevo personal en una organización participan las siguientes áreas: reclutamiento, psicología, servicio médico, capacitación, salarial, y logística. Estas áreas tienen que coordinar entre sí para lograr dicho objetivo. El gerente de Recursos Humanos está preocupado porque muchos supervisores han reclamado por la demora en el ingreso del nuevo personal"

En este caso se identifica la presencia de un equipo de trabajo. Se lograría superar el problema en mención de realizarse una intervención de Desarrollo de Equipo, a través de reuniones entre los representantes de cada área para identificar qué se están realizando bien o mal en relación con su meta, y llegar a acuerdos para la acción.

El Desarrollo de Equipo busca el logro eficaz de objetivos organizacionales, según criterios de cantidad y/o calidad. Indaga la mejor forma de obtener una meta; está más centrado en la tarea. Su interés principal no es analizar procesos internos del equipo (p.ej., comunicación, liderazgo, confianza, motivación).

El caso 1 está vinculado a un equipo de trabajo cuyos miembros pertenecen a áreas laborales que permanecen en el tiempo, pero también puede existir equipos de trabajo temporal que se desactivarían luego de cumplir sus objetivos.

Esta intervención es la más descuidada por los psicólogos al centrarse más en el ámbitode las relaciones interpersonales o estados emocionales de las personas. Para superar dicha tendencia es recomendable retomar los aportes de la Administración de la Calidad (Chang \& Niedzwiecki, 1999), y Reingeniería o mejora de los procesos (Chang, 1996). Ambos enfoques son creaciones de los Administradores de Empresas y en un inicio los psicólogos mostraron mucho interés, pero progresivamente disminuyó su atención. El recuperar dichas aproximaciones ayudaría a centrarnos en la manera que el equipo realiza sus tareas para lograr metas, y además pueden servir de base para construir a partir de ellos. 
En el ámbito de la psicología se ha progresado mucho sobre el enfoque de metas que puede contribuir para el Desarrollo de Equipos. Una de las mejores teorías de motivación y con mayor validación práctica es la Teoría del Establecimiento de Metas (Locke \& Latham, 1990). Esta puede ayudar para una adecuada formulación de metas, identificar las estrategias a utilizar y los factores que influyen en el desempeño, así como las consecuencias a esperar.

\section{CONSTRUCCIÓN DE EQUIPO (Team Building)}

Caso 2. "En una oficina perteneciente a la gerencia de Marketing de una empresa de telecomunicaciones, el personal conformado por 15 trabajadores y un supervisor, presenta muchos conflictos entre ellos. El gerente del área ha solicitado a Recursos Humanos la intervención de los psicólogos para facilitar una mejora del ambiente de trabajo"

Para este caso se requiere la intervención de Construcción de Equipo cuyo objetivo es influir en los procesos internos del equipo según las dimensiones:

- Conductas: Comunicaciones, coordinación, liderazgo, colaboración, toma de decisiones, etc.

- Actitudes: Cohesión, confianza, motivación, compromiso, orientación colectiva, etc.

- Cogniciones: Conocimiento compartido sobre roles de cada miembro, los recursos a utilizar y forma de hacer el trabajo, memoria transactiva, etc.

Para resumir, podemos decir que la Construcción de Equipo está orientada a permitir que un conjunto de personas trabaje eficientemente como equipo, hecho que es posible por sus adecuados procesos internos.

A fin de centrarnos en el análisis de los procesos internos de un "trabajo de equipo" primero debemos tener muy claro su diferencia con el "trabajo de grupo", para ello presentamos a continuación la siguiente situación hipotética en un ámbito académico.

Un profesor solicitó la realización de un trabajo monográfico sobre un determinado tema vinculado a un curso; para ello dividió la clase en grupos. Dos de estos conformados por 4 miembros cada uno presentaron diferentes formas de ejecutar lo solicitado:

- El grupo A acordó que cada miembro realice una síntesis de determinados documentos. Uno de ellos se encargó de recibir los textos de los demás para elaborar los comentarios y sugerencias; finalmente imprimió el documento para entregarlo al profesor.

- El grupo B se reunió para diseñar un esquema del trabajo. Cada uno seleccionó el área que más le interesaba para realizar una síntesis de determinada bibliografía. A través de internet efectuaron un dinámico diálogo crítico sobre lo elaborado individualmente, que permitió realizar ajustes en sus textos. En relación con los comentarios y sugerencias se reunieron e intercambiaron ideas para redactar conjuntamente dichas secciones. Luego un miembro realizó la impresión del documento.

En el caso descrito el grupo A realizó un "trabajo de grupo" y el grupo B un "trabajo de equipo". Este se caracteriza por la mutua influencia entre sus miembros, se produce una especie de sinergia, emerge un resultado que supera los aportes individuales.

Presentemos otro ejemplo para identificar la diferencia entre grupo y equipo. Una familia de 5 miembros compró un sillón, pero este viene en una caja que incluye piezas que requieren ser ensambladas. Retornan a su casa e inmediatamente deciden armarlo; se disputan las piezas y luego cada uno cree haber integrado una parte. Intentan juntarlas, pero sucede que han acoplado mal las piezas de manera que no aparece lo esperado. Especulan que faltan piezas o existe fallas de fábrica. Después de esta frustración el padre solicita llegar a un acuerdo sobre la mejor forma de lograr el objetivo; deciden analizar detenidamente las instrucciones incluidas en la caja, luego se identifique las distintas partes que serían armadas de manera secuencial hasta obtener el mueble final. Este procedimiento les permitió tener éxito al facilitar el trabajo de equipo. 
Una vez detectada la presencia de un trabajo de equipo debemos centrar nuestra atención en sus procesos internos. Así tenemos en el caso del grupo B de la tarea académica destacan: planificación, distribución de roles, y coordinación. Para el caso del sillón tenemos: liderazgo del padre, plan de trabajo, análisis de la tarea, y coordinación. El tener identificados los procesos estaremos en condición de indagar su calidad y en qué medida requieren modificarlos o reforzarlos. También es posible que un equipo de trabajo presente un bajo desempeño por la carencia de determinado proceso, lo cual implicará implementarlo.

En la labor del psicólogo nos encontramos con trabajos de equipo fácilmente identificable como es el caso del psicólogo clínico que realiza terapia grupal, quien debe estar alerta a los procesos que generalmente están presentes tales como: liderazgo, conflictos, apoyos, confianza, participación, motivación. Realmente son dimensiones emergentes que se desarrolla en el equipo conforme avanza el tratamiento. Se puede afirmar que el psicólogo debe ser experto para fomentar un trabajo de equipo que facilite la terapia

Normalmente en las organizaciones se requiere considerar diversos equipos relacionados para lograr un determinado objetivo. $\mathrm{Al}$ respecto podemos mencionar que el presente documento en cierta medida es resultado de un trabajo de equipo. Como integrantes podemos ubicar al autor, familiares, colegas, coordinador e informantes oficiales; a través de revisiones y diálogos iterativos se logró la versión final del documento. Pero además existe otro equipo que tendrá la responsabilidad de conseguir la impresión de la revista en la que aparezca el artículo.

Generalmente se observa que los alumnos en las universidades están habituados a realizar un trabajo de grupo, pero en la actividad profesional se exige más el trabajo de equipo. El problema en parte es responsabilidad de los docentes que no otorgan la debida importancia al trabajo de equipo del alumnado, ni se recompensa el esfuerzo que puedan realizar con dicho fin. Se requiere adquirir competencias que permita a un miembro ser útil para el buen desempeño de su equipo. Una ayuda para este fin podría ser el incremento de la capacidad para detectar la presencia del trabajo de equipo y sus procesos internos en situaciones cotidianas en las que participemos. Por ejemplo, al asistir a un restaurant encontramos, recepcionista, mozo, cocinero, cajera; la satisfacción del cliente dependerá mucho si estos miembros trabajan como un buen equipo. Si observamos con detenimiento el proceder de los equipos se fortalecería la aptitud para diagnosticar estos contextos.

\section{ENTRENAMIENTO DE EQUIPO (Team Training)}

Caso 3. "Durante el gobierno de Alberto Fujimori el 17 de diciembre de 1996 catorce terroristas del MRTA ingresaron violentamente a la residencia del embajador de Japón en Lima, cuando se realizaba una cena por una festividad japonesa. Todos los invitados fueron capturados como rehenes. Después de 126 días que duró la ocupación de la residencia, Fujimori dispuso una intervención militar para liberar a los rehenes, para ello se conformó previamente un equipo militar especial, cuyos miembros practicaron sus acciones con el asesoramiento de expertos, utilizando una réplica de la residencia construida para dicha operación. El día 22 de abril del 1997 se procedió con el rescate de los rehenes empleando un túnel recién construido debajo de la casa. En el operativo fallecieron 1 rehén, 2 militares, y todos los terroristas".

En este caso existe un ejemplo típico de Entrenamiento de Equipo, donde los miembros aprenden y practican sus actividades antes de proceder a ejecutarlo en la realidad. Otro caso del mismo tipo puede presentarse en el ámbito médico para la realización de alguna intervención quirúrgica, teniendo como miembros al cirujano, enfermera, anestesiólogo y personal auxiliar. Un tercer ejemplo lo observamos en el entrenamiento de una tripulación responsable de la conducción de una aeronave.

Desde el punto de vista metodológico el entrenamiento de equipo se sustenta en los mismos principios y enfoques existentes para el entrenamiento de personal que ejecutará su trabajo de manera individual (Salas et al,2015). Por este motivo, el psicólogo interesado en aplicar esta intervención requiere tener una formación en capacitación y entrenamiento. 
En el ámbito escolar se presenta diversas situaciones en la que está presente el trabajo de equipo, así por ejemplo cuando se reúnen profesor, psicólogo, y padre de familia para analizar determinadas conductas de algún alumno; o un equipo que organiza una actividad académica. Existe al respecto programas de entrenamiento como el Teach Teamwork patrocinado por la APA para fomentar el buen desempeño de los equipos de trabajo en el ámbito escolar, a través de los módulos: comunicación, monitoreo de la situación, apoyo mutuo, y liderazgo. A este programa se puede acceder en: https://www.apa.org/education/k12/teachteamwork (Benishek et al., 2016).

En los centros de cuidado de salud (p.ej., clínicas, hospitales) los psicólogos clínicos pueden contribuir al participar como miembro de un equipo orientado a disminuir los errores médicos que regularmente se presenta en dichos contextos. Las intervenciones de entrenamiento de equipo han mostrado ser útiles para dicho fin (Buljac-Samardzic et al., 2020; Rosen et al., 2018; Edmondson, 2012). También puede diseñar y ejecutar programas utilizando principios y metodologías de la ciencia del trabajo de equipo, como lo demuestra de manera detallada Traylor et al. (2021) para el manejo adecuado de las reacciones conductuales y emocionales del personal médico que atiende a pacientes afectados con covid-19.

En la labor de los psicólogos sociales también está presente la necesidad de implementar entrenamiento con fines de mejora de los equipos de trabajo. No requiere limitarse a un solo contexto al existir diferentes entidades involucradas (p.ej., hogar, sindical, gubernamental, barrial).

Respecto a la labor del psicólogo organizacional no se menciona algo especifico, a razón que el autor de este trabajo es de dicha especialidad, quién en el texto presenta situaciones que pueden sugerir al lector posibles labores a realizar.

\section{COMPLEMENTARIDAD DE LAS INTERVENCIONES}

No son excluyentes los tres tipos de intervención, puesto que según la situación se puede utilizar más de uno. Por ejemplo, si un equipo no logra sus metas por falta de confianza entre sus miembros, una intervención de Construcción de Equipo ayudaría a superar el problema. También podría darse el caso que, en la tripulación de una aeronave puede estar presente algún conflicto interpersonal que esté interfiriendo con una buena coordinación entre ellos.

Sugerimos que en la práctica el consultor considere primero a los equipos de trabajo desde la perspectiva de los clientes y procesos laborales (Desarrollo de Equipo). De identificarse problemas para lograr sus metas por fallas en sus procesos internos, entonces se justificaría considerar la Construcción de Equipo.

Es bueno señalar que, en ciertas circunstancias se justificaría realizar Construcción de Equipo sin estar relacionado directamente con el rendimiento laboral. Por ejemplo, el lograr una mejora en el clima laboral podría favorecer la satisfacción de los miembros del equipo sin afectar su desempeño; rendirían igual a pesar de estar más contentos y felices que antes de la intervención. Es bueno agregar que, consideramos de igual valor la salud psicológica y el rendimiento laboral.

Actualmente en nuestro medio el psicólogo mayormente realiza talleres o cursos para el Desarrollo de Equipo que incluyen actividades novedosas y entretenidas, pero generalmente sin buscar nexos claros con el rendimiento laboral. Lograr un "equipo feliz" no implica que mostrará un buen rendimiento en el trabajo (Tannenbaum \& Salas, 2021).

Un aspecto generalmente descuidado en el ámbito de la capacitación grupal, son algunos efectos secundarios no vinculados directamente a los objetivos explícitos de la intervención formativa. Entre ellos podemos mencionar los siguientes:

- El abandonar temporalmente la labor rutinaria podría permitir a los participantes disminuir niveles de estrés o aburrimiento.

- Conocer personalmente a compañeros de labores o de otras organizaciones. Permitir el desarrollo de redes sociales.

- Percibir el interés de la organización por su personal. 
- Adquirir alguna habilidad o conocimiento que puede ser útil personalmente o para otro contexto (p.ej., familiar, amical)

- Aprender de las experiencias e intervenciones de los demás participantes

El psicólogo debería acordar con la administración que autorizaría la intervención, si el objetivo es contribuir para el logro de metas organizacionales (Desarrollo de Equipo) o fortalecer los procesos internos del equipo (Construcción de Equipo). En este último caso, debe estar bien definido si el objetivo está o no vinculado directamente al rendimiento laboral, y si merece destacar determinados efectos secundarios del proceso formativo. Para el entrenamiento de equipo quizás será más fácil llegar a un acuerdo sobre metas.

\section{ORIENTACIONES BÁSICAS PARA UNA ADECUADA INTERVENCIÓN}

\section{Identificar la necesidad de intervenir a nivel de equipo}

Se requiere estar convencido que la intervención es a nivel de equipo (Thayer et al., 2014). Existe un equipo de trabajo si dos o más personas tienen que interactuar para lograr "...objetivos específicos, compartidos y valorados" (Diaz Granados \& Salas, 2008, p.14). Podría presentarse problemas entre personal no interdependiente y sin involucrar a los demás, en cuyo caso sería lo más aconsejable una intervención a nivel individual.

\section{Manejo del Pensamiento de Equipo en la intervención grupal}

El fenómeno del Pensamiento de Equipo está presente cuando los miembros poseen mucho respecto entre sí, evitan considerar aspectos negativos, están convencidos que ellos poseen la "verdad", y llegan fácilmente a decisiones sin realizar un adecuado análisis. Esto puede ser superado desde diferentes perspectivas tales como: fomentar la "seguridad psicológica" en el equipo (Edmondson, 2018) de manera que los participantes puedan expresar sus verdaderas apreciaciones; el facilitador manifiesta que desempeñará el rol de "abogado del diablo" frente a las opiniones de ellos, y que su intención realmente no es molestar; explicar en qué consiste el Pensamiento de Equipo y sus consecuencias negativas, presentando algunos ejemplos (p.ej.,Intervención de John Kennedy para invadir Cuba; Janis, 1972).

\section{Utilizar descripciones antes que evaluaciones en la comunicación}

Es muy útil preferir el uso de descripciones y evitar el modo evaluativo durante la comunicación, especialmente en situaciones de conflicto. $\mathrm{Al}$ respecto, mencionaremos una intervención que el suscrito y otro colega realizamos en una organización, en la que existía un área con muchos conflictos entre supervisor y personal. Se propuso al gerente que sería conveniente realizar reuniones grupales entre los involucrados. El gerente rechazó la propuesta "porqué se iban matar entre ellos". Insistimos con un mayor sustento. El gerente aceptó el pedido, pero dejó en constancia que "si se empeoraba la situación sería exclusiva responsabilidad nuestra”.

En las dinámicas grupales se estableció que en las intervenciones se debe utilizar únicamente afirmaciones descriptivas y estaban prohibidas las de tipo evaluativo. Para precisar las diferencias entre los conceptos, presentamos un caso hipotético de un "trabajador que al salir de la oficina cerraba la puerta con demasiada fuerza que hacía vibrar las lunas" (descripción), y al día siguiente su supervisor le indicó que es un "patán y mal educado al salir de la oficina" (evaluación).

Efectuamos las reuniones grupales con estricta aplicación de la principal regla de comunicación que permitió abordar de manera más objetiva los problemas interpersonales y disminuyó las intervenciones defensivas. Finalmente se logró establecer acuerdos orientados a facilitar en lo sucesivo su relación. Posteriormente con propósito de seguimiento nos reunimos con el gerente del área, quien indicó que el supervisor y su personal estaban trabajando bien, y agregó que por "algo somos psicólogos para evitar una matanza anunciada".

\section{equipo \\ Promover el aprendizaje en el trabajo de}

Todo cambio implica la presencia de aprendizaje. Podemos afirmar que el cambio y el aprendizaje son dos caras de una misma moneda. En la intervención 
para fomentar un eficiente trabajo de equipo, se debería tener presente la dimensión del aprendizaje con sus componentes básicos: diagnóstico, diseño, acción, y reflexión (Edmondson, 2012; Solf, 2014). A través de la reflexión se permite identificar qué se hizo bien o mal, y determinar acciones futuras. Sin reflexión la experiencia sería de poca utilidad. El aprendizaje debería estar centrado cómo se realiza el trabajo y sobre los procesos internos del equipo.

\section{Evitar el Error de Atribución Fundamental}

Este sesgo está presente cuando en el diagnóstico se considera al personal involucrado como los principales y a veces únicos responsables de la situación, sin considerar los factores contextuales. Se puede llegar a pensar que el problema existe por la falta de motivación, personalidad inadecuada, etc. de los miembros y no se examina las características de las tareas, el tipo de supervisión, la cultura de la organización, u otra dimensión pertinente. Si bien es importante las características individuales, también merece considerar los entornos próximo y distante. La formación de los psicólogos centrada más en la persona tiende a propiciar este sesgo.

\section{CONSTRUCCIÓN DE EQUIPOS VIRTUALES}

Si bien este trabajo no tenía como motivo indagar sobre los equipos virtuales, es bueno señalar algunos aspectos. Así tenemos que el confinamiento como una forma de protección frente al covid-19 originó el incremento del teletrabajo, y dentro de esta modalidad se ubica la utilización de los equipos virtuales. Se pronostica que esta forma de trabajo continuará en mayor medida. Para los psicólogos es una oportunidad para investigar más esta área; a pesar de que existe alguna investigación es necesario encontrar respuestas a muchas interrogantes. Sobre los equipos virtuales es indispensable adquirir conocimientos sobre aspectos como:

- ¿Qué problemas de comunicación presentan?

- ¿Qué tipo de liderazgo requieren?

- ¿Cómo funcionan los mecanismos motivacionales?

- ¿De qué manera influyen las diferencias individuales?
- ¿Cómo pueden coordinar mejor diversos equipos virtuales conformado por diferentes especialistas?

- ¿Cómo se evaluaría su desempeño?

- ¿Qué relación existe entre pertenecer a equipos virtuales y el compromiso e identificación con la organización?

- ¿Cómo obtener sinergias entre equipos virtuales y presenciales?

Para fomentar la Construcción de Equipos Virtuales no será suficiente las actividades y juegos que actualmente proliferan en la web. Se requiere una aproximación orientada por teorías y modelos que faciliten la investigación científica.

\section{SUGERENCIAS Y CONCLUSIONES}

A los interesados en continuar aprendiendo sobre el tema recomendamos el libro Teams that Work de los psicólogos Scott Tannenbaum y Eduardo Salas (2021). Es un libro muy práctico, de agradable lectura, y excelente síntesis de más de 30 años de investigación científica. Está dirigido principalmente a miembros de organizaciones laborales, como gerentes, supervisores, consultores, miembros y líderes de equipos, etc. Esta característica permitiría a los psicólogos disponer de un lenguaje común con dichos miembros, quienes utilizarían el libro al seguir estudios de MBA o cursos específicos (p.ej., liderazgo, trabajo de equipo, coaching). Podemos afirmar que Teams that Work es actualmente el mejor libro de psicología organizacional. Además de este libro sugerimos los aportes de Amy Edmondson $(2012,2018)$ profesora de la Universidad de Harvard.

Podemos concluir que en la labor del psicólogo de todas las especialidades está presente la necesidad de tener experticia para contribuir con el eficaz trabajo de equipo. Esto requiere básicamente tener interés por adquirir una formación en el tema a través de cursos, seminarios, talleres, lecturas, etc. De igual manera es deseable que los centros de instrucción superior otorguen la debida importancia a esta área, que actualmente es considerada una ciencia que muestra un continuo desarrollo. 
A continuación, en la Sección Anexos se presenta algunos ejemplos y recursos vinculados a las tres intervenciones, para ayudar a identificar con mayor precisión sus diferencias, siendo este un objetivo del documento.

\section{REFERENCIAS}

Aguilar, R.A., de Antonio, A. (2005). Entrenamiento de equipos: Una estrategia asistida por entornos virtuales inteligentes. Comunicaciones, 2, 25-33

Belbin, R. M. (1993) Team roles at work, Butterworth Heinemann: Oxford.

Benishek, L.E., Gregory, M.A., Hodges, K., Newell, M., Hughes, A.M., Marlow, S., ...Salas, E. (2016). Bringing the science of team training to school-based teams. Theory Into Practice, 55(2), 112-119, DOI: 10.1080/00405841.2016.1148987

Biech, E. (2007). 90 World-class activities by 90 world-class trainers. John Wiley \& Sons.

Biech, E. (Ed.) (2011). The book of road-tested activities. San Francisco, CA: John Wiley and Sons

Blickensderfer, E., Cannon-Bowers, J.A., \& Salas, E. (1998). Cross-training and team performance. En J.A. Cannon-Bowers \& E. Salas (Eds.) Making Decisions Under Stress: Implications for Individual and Team Training (pp.299-311). APA Press

Buljac-Samardzic, M., Doekhie, K.D., \& van Wijngaarden, J.D.H. (2020). Interventions to improve team effectiveness within health care: a systematic review of the past decade. Human Resource for Health, 18(2), https://doi. org/10.1186/s12960-019-0411-3

Chang, R.Y. (1996). Mejora Continua de Procesos. Granica S.A.

Chang, R.Y., \& Niedzwiecki, M.E. (1994). Las herramientas para la mejora continua de la calidad (Vol.I). Granica S.A.

DiazGranados, D., \& Salas, E. (2008). Temas emergentes y conclusiones en la investigación acerca de los equipos y el trabajo de equipo. Persona, 11, 11-32

DiazGranados, D., \& Salas, E. (2010). Desarrollo de la literatura de entrenamiento individual y de equipo. Persona, 13, 43-69

Driskell, T., Driskell,J.E., Burke, C.S.,\& Salas. (2017). Team roles: A review and integration. Small Group Research. DOI: 101177/104649417711529

Edmondson,A.C.(2012). Teaming: How organizations learn, innovate, and compete in the knowledge economy. Jossey-Bass

Edmondson, A.C. (2018). The Fearless Organization: Creating Psychological Safety in the Workplace for Learning, Innovation, and Growth. Wiley

Folwlkes, J., Dwyer, D.J., Oser, R. L., \& Salas, E. (1998). Event-Based Approach to Training (EBAT). The International Journal of Aviation Psychology, 8(3), 209-221

Glanz, B.A. (2007). Commonalities and uniquenesses. En E. Biech (Ed.) 90 World-class activities by 90 world-class trainers (pp. 256-258). John Wiley \& Sons.

Goldsmith, M. (2009). Try Feedforward instead of Feedback. En E. Biech (Ed.) The 2009 Pfeiffer Annual Training (pp. 175-180). Pfeiffer

Goldstein, G. (2004). My worst nightmare. En E. Biech (Ed.) The 2004 Pfeiffer Annual Training (pp. 93-96). Pfeiffer

Gray, D., Brown, S., \& Macanufo, J. (2012). Gamestorming: 83 juegos para innovadores, inconformistas y generadores del cambio. Ediciones Deusto

Janis, I. L. (1972) Victims of groupthink, Houghton Mifflin

Kozlowski, W.J. (1998). Training and Developing Adaptive Teams: Theory, Principles, and Research. En J.A. Cannon-Bowers \& E. Salas (Eds.). Making Decisions Under Stress: Implications for Individual and Team Training (pp.115-153). APA Press

Locke, E.A., \& Latham, G. P. (1990). A Theory of Goal Setting \& Task Performance. Prentice Hall 
Pagano, K. O. (2013). Immersive Learning: Designing for authentic practice. Alexandria. ASTD Press

Paoletti, J., Kilcullen, M.P., Salas, E. (2021). Teamwork in Space Exploration. En L.B. Landon, K.J. Slack, \& E. Salas (Eds.). Psychology and Human Performance in Space Programs: Research at the frontier (pp. 195-216). CRS Press

Reyes, D., Tannebaum, S.T., \& Salas, E. (2018). Team Development: The power of Debriefing. People + Strategy, 41 (2), 46-51

Rosen, M.A., Salas, E., Wu, T.S., Silvestri, S., Lazzara, E.H, Lyons, R, Weaver, S.J., \& King, H.B. (2008). Promoting teamwork: An event-based approach teamwork to simulation-based teamwork training for emergency medicine residents. Academic Emergency Medicine, 15, 1-9

Rosen, M.A., DiazGranados, D., Dietz, A.S., Benishek, L.E., \& Weaver, S.J. (2018). Teamwork in Healthcare: Key Discoveries Enabling Safer, High-Quality Care. American Psychologist, 73(4), 433-450

Salas, E., Dickinson, T.L., Converse, S.A. and Tannenbaum, S.I.(1992), Toward an understanding of team performance and training. En R.W. Swezey, R.W., \& E. Salas, E. (Eds). Teams: Their training and Performance (pp. 3-29). Praeger

Salas, E., \& Cannon-Bowers, J.A. (1997). Methods, Tools, and Strategies for Team Training. In M. A. Quiñones \& A. Ehrenstein (Eds.), Training for a rapidly changing workplace: Applications of psychological research (pp. 249-279). APA Press

Salas, E. et al. (2015). Team Training Essentials: A Research-Based Guide. Routledge

Fecha de recepción: 26 de abril 2021

Fecha de aceptación: 9 de setiembre 2021
Salas, E., Tannenbaum, S., Kozlowski, S.W.J., Miller, C.A., Mathieu, J.E., \& Vessey, W. B. (2015b). Teams in space exploration: A new frontier for the science of team effectiveness. Current Directions in Psychological Science, 24(3), 200- 207

Salas, E., Rico, R., \& Passmores, J. (2017). The Wiley Blackwell Handbook of the Psychology of Team Working and Collaborative Processes. Wiley Blackwell

Silberman, M. (2010). Unforgettable Experiential Activities. Pfeiffer

Smith-Jentsch, K.A., Zeisig, R.L., Acton, B., \& McPherson, J. A. (1998). Team dimensional training: A strategy for guided team self-correction. En J.A. Cannon-Bowers \& E. Salas (Eds.) Making Decisions Under Stress: Implications for Individual and Team Training (pp. 271-297). APA Press

Solf, A. (2014). El psicólogo organizacional como facilitador del aprendizaje: Su principal rol. Avances en Psicología, Unifé, 22(1), 29-48

Tannenbaum, S., \& Salas, E. (2021). Teams that Work: The seven drivers of team effectiveness. Oxford University Press.

Thayer, A.L., Rico, R., Salas, E., \& Marlow, S.L. (2014). Teams at Work. En M. C. W. Peeterrs, J. de Jonge., \& T. W. Taris (Eds.). An introduction to contemporary work psychology (pp. 434-457). Wiley Blackwell

Traylor, A.M., Tannenbaum, S.I., Thomas, E., J., \& salas, E. (2021). Helping Healthcare Teams Save Lives During COVID-19: Insights and Countermeasures From Team Science. American Psychologist, 76(1), 1-13. 


\section{ANEXOS}

\section{DESARROLLO DE EQUIPO}

A continuación, se incluyen algunos esquemas que podrían orientar la labor de un consultor interesado en apoyar a los equipos de trabajo para la consecución de sus metas:

1.1. Mejorar el aporte de los equipos a la organización. Los miembros de un equipo de trabajo se reúnen para decidir acciones orientadas a incrementar su rendimiento en relación con las metas organizacionales específicas. La indagación podría orientarse según lo siguiente:

- ¿Qué aspectos de nuestro trabajo estamos desempeñando bien?

- ¿Qué aspectos de nuestro trabajo requiere una mejora? ¿Por qué no podemos avanzar?:

- ¿Barreras para nuestro trabajo?

- ¿Carencias de habilidades / Conocimientos / Experiencia?

- ¿Forma de realizar el trabajo de equipo?

- ¿Carencia de apoyos?

- ¿Metas no claras o falta de consenso?

- ¿Inadecuada supervisión?

- ¿Carencia de claros indicadores para medir avances?

- ¿Los roles laborales no están claros para los miembros?

- ¿Qué puede aportar cada miembro para una mejora?

- ¿Qué ayudas de los demás desearían los miembros del equipo?

- ¿Qué acciones están dispuestos a realizar como equipo?

- ¿Qué decisiones adoptarían?

1.2. Aporte del equipo a los clientes. Los equipos de trabajo poseen objetivos a lograr para satisfacer determinadas necesidades de los clientes internos (e.d., otras áreas de la empresa) o externos (e.d., fuera de la empresa). Desde esta perspectiva el equipo puede efectuar indagaciones tales como:

- ¿Quiénes son nuestros clientes?

- ¿Cuáles son las principales necesidades de los clientes?

- ¿Qué servicios/recursos proporcionamos para satisfacer las necesidades del cliente?

- ¿Qué criterios -tiempo, costos, calidad, etc.- utilizan los clientes para evaluar el aporte de nuestro equipo?

- ¿Cuán satisfecho suponemos que están los clientes con nuestro aporte?

- ¿En qué servicios/recursos proporcionados están más satisfechos nuestros clientes?

- ¿Cómo podemos mejorar la entrega de servicios/recursos a nuestros clientes?

\subsection{Analizar y mejorar el proceso de trabajo:}

- ¿Los objetivos son los adecuados? ¿Están bien formulados?

- ¿Todas las tareas establecidas son necesarias? ¿Algunas deben ser eliminadas o modificadas?

- ¿La secuencia de las actividades es adecuada?

- ¿Los tiempos utilizados para las tareas están bien establecidos?

- ¿Cuáles son los criterios para medir los resultados? ¿Cómo proceder con la medición?

- ¿Qué decisiones se adoptarían? 
1.4. Utilizar herramientas de mejora de la calidad: Por ejemplo: Diagrama de Afinidad, Análisis de Campo de Fuerzas, Diagrama de Causa y Efecto, Diagrama Matricial (Chang \& Niedzwiecki, 1994; Brown, \& Macanufo, 2012).

1.5. Reflexión después de la acción (Debriefing). Reyes, Tannenbaum y Salas (2018) sugieren las siguientes acciones que un supervisor o facilitador de un equipo de trabajo puede realizar para resaltar los aprendizajes a partir de la experiencia.

Solicitar a los miembros del equipo que indiquen:

- ¿Qué sucedió?

- ¿Qué hicimos bien? ¿Qué desafíos enfrentamos?

- ¿Qué deberíamos hacer diferente o considerar la próxima vez?

- ¿Qué podría ayudarnos para ser más efectivo? ¿Qué necesitamos?

- Añadir sus observaciones/recomendaciones y confirmar percepciones de los miembros.

- Resumir y llegar acuerdos

\section{CONSTRUCCIÓN DE EQUIPO}

Se tiene como objetivo central influir en los procesos internos del equipo de trabajo para superar limitaciones o carencias, así como optimizar lo existente. Con dicha finalidad se realiza actividades específicas según determinada perspectiva. A continuación, se incluyen algunos ejemplos de intervención desde el enfoque de capacitación, indicando el objetivo de interés:

2.1. Fomentar un mayor conocimiento entre los miembros (Glanz, 2007): Conformar subgrupos cuyos miembros identifiquen características comunes a todos (p.ej., estudiantes, nacidos en provincia). Continuar el diálogo para descubrir de cada participante una característica única, esto es, que solo un miembro la posee y los demás no (p.ej., hablar ruso). Luego, divulgar los resultados de cada subgrupo al total del salón, mencionando en primer lugar las semejanzas, y en el caso de las diferencias comunicarlo sin mencionar nombres, de manera que los oyentes intenten adivinar a quien corresponde cada singularidad. Finalmente, efectuar una discusión y análisis general.

2.2. Aprender de experiencias negativas (Goldstein, 2004): Formar subgrupos para que cada participante comparta una experiencia muy difícil que haya tenido con otra persona, por ejemplo, un supervisor con un subordinado, un vendedor con un cliente, o un alumno con su profesor. Que describa en qué consistió el problema, cómo se originó, qué acciones realizó, y resultados conseguidos. Luego cada subgrupo informa al salón las mejores experiencias. La cantidad de información dependerá del tiempo disponible y del número total de participantes. Realizar análisis y comentarios pertinentes.

2.3. Adquirir nuevas perspectivas (Feedforward; Goldsmith, 2009): Esta actividad es útil para adquirir nuevas perspectivas que permitan la solución de un problema o implementar cambios. Se obtiene mejores resultados cuando los participantes son muy diferentes en cuanto a experiencia o formación profesional. Por ejemplo, en un taller sobre la administración del tiempo, los participantes pueden desear efectuar cambios específicos para no procrastinar en su trabajo. Se fomenta el diálogo entre parejas de participantes: inicialmente uno de los dos explica cuál es su problema; el otro le proporciona sugerencias para superar el problema. El receptor de las sugerencias escucha, toma notas, y no hace ningún comentario. Luego se invierten los roles. Al finalizar ambos buscan otros interlocutores, y así prosiguen hasta que el tiempo disponible lo permita. Luego realizar una discusión general. 
2.4. Desarrollo de la creatividad (Silberman, 2010): Esta actividad se orienta a demostrar cuan creativa puede ser las personas para utiliza recursos limitados, y favorecer la cohesión de equipo. Dividir a la clase en subgrupos de cuatro a seis miembros. Entregarle a cada equipo un juego de tarjetas o fichas (mejor si son de diferente tamaño) y plumones de colores. Indicarles que traten de ser lo más creativo posible para construir la "casa de sus sueños" utilizando solo las tarjetas. Pueden doblar, cortar, y dibujar sobre las tarjetas. No se permite utilizar ningún otro recurso. Otorgar al menos 15 minutos para la construcción. No presionar a los equipos para que tengan una experiencia exitosa. Cuando han finalizado la construcción, proceder con un "tour" por el "barrio", de manera que los diversos equipos presenten y expliquen su trabajo a los demás. Aplaudir por la labor de cada equipo. Fomentar la reflexión con todos los participantes: ¿se han sorprendido cómo usted y otros pudieron crear con recursos limitados? ¿qué ayudó o impidió la labor del equipo? ¿cuál ha sido el clima de trabajo? ¿existió algún líder? ¿cómo evaluaría el trabajo de su equipó? ¿qué ha aprendido que le puede servir para su trabajo?

2.5. Análisis del liderazgo (Biech, 2011): Esta actividad permitiría a los participantes adquirir conciencia sobre su estilo de liderazgo e identificar las principales influencias formativas. El facilitador explica los objetivos de la sesión y entrega un cuestionario para que respondan individualmente las siguientes preguntas:

- ¿Qué lugar de nacimiento ocupa entre sus hermano(a)s?

- ¿En su niñez, en qué juegos generalmente participaba? ¿Usualmente era líder, seguidor, o un poco de ambos?

- Mencione a sus mejores amigos con los que creció. ¿Especialmente que le agradó de cada uno de ellos?

- Nombre a una persona cercana con quién usted creció (padre u otro adulto). ¿Qué cualidades de liderazgo dicha persona exhibía?

- Nombre un profesor quién tuvo una fuerte influencia en usted. ¿De qué manera esta persona influyó en usted?

- ¿Cuál fue su primer trabajo "real”? ¿Qué aprendizajes de liderazgo obtuvo de este trabajo?

- ¿Quién fue su mejor jefe? ¿De qué manera esta persona fue un buen líder?

- ¿Quién fue su peor jefe? ¿De qué manera esta persona fue un mal líder?

Luego de finalizar con el cuestionario, formar subgrupos en el cual cada miembro comunica sus respuestas. Fomentar los mutuos comentarios y la retroinformación. Al finalizar, cada subgrupo informa a toda la clase los resultados más importantes del diálogo realizado. El facilitador proporcionará observaciones pertinentes.

2.6. Identificar los roles desempeñados por los miembros del equipo: Belvin (1993) identificó determinados roles necesarios que deberían desempeñar los miembros de un equipo. Recientemente Driskel et al. (2017) realizaron una investigación más amplia sobre los roles que los diferentes miembros desempeñan al participar en un equipo de trabajo. Identificaron roles favorables y algunos desfavorables. A continuación, se presentan dichos roles que puede ser útil para que los miembros adquieran conciencia de cual(es) normalmente expresan en sus actividades:

- Líder de equipo.- Guía y controla. Facilita las actividades, trae orden, estructura la tarea, actúa como guía para el logro de la tarea, organiza y coordina.

- Impulsador.- Estimula al grupo para seguir adelante. Proporciona ánimo y energía al equipo para superar dificultades y lograr las metas.

- Buscador de poder.- Busca autoridad o superioridad, interrumpe las contribuciones de otros, intenta obtener control, se opone al líder o es agresivo hacia los demás. 
- Cohesionador.- Alivia la tensión, media en los desacuerdos, elogia de manera sincera, se muestra solidaria(a) y entusiasta, apoya a los demás, mantiene la moral, promueve la cohesión grupal.

- Coordinador.- Coordina actividades entre los miembros de los subgrupos, clarifica las relaciones según las tareas, mantiene abierto los canales de comunicación, facilita la participación de otros, y promueve ideas dentro y fuera del equipo.

- Crítico.- Típico rol del "defensor del diablo". Discrepa y se opone, muestra cinismo, tiende a ir contra el resto del equipo, y puede lograr que los demás se comporten a la defensiva.

- Seguidor.- Participa en las discusiones grupales y decisiones, construye sobre las ideas de los demás, efectivo oyente y facilitador, acepta las asignaciones, busca cooperación, evita los desacuerdos.

- Apoyo al trabajo de equipo.- Planea la implementación de las acciones, realiza registros de los procesos y el rendimiento del equipo, toma iniciativas para asegurar el éxito del grupo, prepara las reuniones del equipo y clarifica, y ejecuta acciones que contribuyan al logro de las metas del equipo.

- Evaluador.- Incluye conductas relacionadas a la inspección y evaluación. Analiza y evalúa propuestas, es cuidadoso y meticuloso, focalizado en hechos y datos vinculados a las tareas.

- Buscador de atención.- Intenta conseguir la atención y simpatía del equipo, espera que otros hagan el trabajo, aprovecha para expresar puntos de vista personal.

- Buscador de información e ideas.- Indaga o proporciona opiniones; investiga para clarificar; ofrece hechos e información; señala inconsistencias y las clarifica; sintetiza nuevas ideas y presenta soluciones.

- Finalizador de tarea.- Realiza conductas rutinarias orientadas a culminar la tarea, centrado en el detalle.

- Distante.- Muestra tensión, contribuye poco, se lamenta y queja, disminuye el espíritu de equipo, percibe el esfuerzo como una pérdida de tiempo, predomina una actitud de "no puede hacerse"

\section{ENTRENAMIENTO DE EQUIPO}

El entrenamiento ha evolucionado desde un enfoque sin sustento teórico hasta la actualidad que es considerado una ciencia útil para individuos y equipos (Salas et al., 2015; Salas \& Cannon-Bowers,1997). A continuación, se presenta algunos tópicos vinculados al entrenamiento de equipo.

3.1. Entrenamiento cruzado: A los miembros del equipo se les capacita sobre los diferentes roles y funciones de los demás. Esta formación puede ser de manera global, específica, o en todas las funciones según el rol. Por ejemplo, a las enfermeras se les proporciona información sobre la labor del cirujano. Esto facilitará las coordinaciones cuando ambos participen en un equipo responsable de una intervención quirúrgica (Blickensderfer et al.,1998)

3.2. Desarrollo de la memoria transactiva: Cada miembro conoce de los demás qué habilidades e información poseen; es una especie de memoria colectiva de la cual se puede acceder a dichos recursos según las necesidades del equipo (Edmondson, 2012).

3.3. Entrenamiento de equipos autocorrectivos: Orientado a permitir que los miembros de un equipo logren corregir sus competencias sin la ayuda de un instructor externo. Expresan lo que ocurrió tanto lo esperado como lo inesperado. A través de la reflexión y la autocrítica detectan problemas específicos y determinan acciones correctivas (Kozlowski, 1998; Smith-Jentsch et al., 1998; DiazGranados \& Salas, 2010).

3.4. Entrenamiento de equipo utilizando simulaciones: Como es el caso de utilizar maniquíes especiales que permitan a un equipo realizar "intervenciones quirúrgicas" en un proceso de aprendizaje. Otra estrategia es el entrenamiento en un ambiente diferente a un salón de clase, como puede ser un ambiente campestre donde el 
equipo tiene que lograr un objetivo establecido por el facilitador, manteniendo similitudes psicológicas con el trabajo real (Outdoor Training).

3.5. Aplicación del Aprendizaje Inmersivo (Immersive Learning; Pagano, 2013): Se orienta a permitir que los miembros utilicen la tecnología actual para practicar lo aprendido en una capacitación. Por ejemplo, un equipo después de haber concluido una capacitación sobre liderazgo, pueden participar en mundos virtuales (p.ej., Second Life) para practicar lo aprendido. También puede utilizarse para dicho fin, juegos de consola, tecnologías móviles, realidad aumentada, plataforma de medios sociales, herramientas en la web, etc. En la medida que la tecnología avance se dispondrá de mayores recursos. La utilización de la tecnología para practicar antes de aplicarlo en la realidad permite que los errores cometidos en dicha etapa tengan efectos sólo a nivel de aprendizaje.

3.6. Entrenamiento de equipo basado en eventos (Event-Based Approach to Training, EBAT) Este enfoque se caracteriza por establecer una relación muy congruente entre los diferentes estadios del entrenamiento: Identificar competencia a desarrollar- Objetivos del entrenamiento- Identificar los KSAs (conocimientos, habilidades y actitudes)- Establecer contexto o escenario- Presentación de eventos activadores en el contextoRespuesta del participante- Medición desempeño- Retroinformación- Acciones futuras. Los eventos se exponen de acuerdo con las facilidades disponibles: simuladores, programas en computador, representación de roles, etc. El EBAT posee una orientación conductual; identifica cuidadosamente los eventos necesarios para activar respuestas adecuadas según KSAs y objetivos de entrenamiento. Es adaptable a diferentes contextos -p.ej., aviación, militar, salud- (Fowlkes et al., 1998; Rosen et al., 2008) 